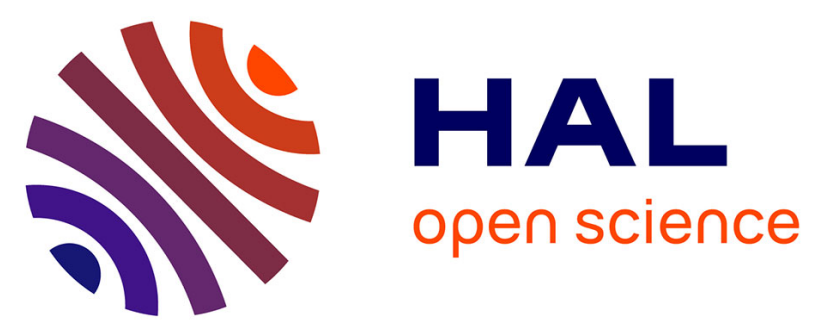

\title{
Vector ordering and multispectral morphological image processing
}

\author{
Santiago Velasco-Forero, Jesus Angulo
}

\section{To cite this version:}

Santiago Velasco-Forero, Jesus Angulo. Vector ordering and multispectral morphological image processing. Vector Ordering and Multispectral Morphological Image Processing, 11, pp.223-239, 2014, Advances in Low-Level Color Image Processing, 10.1007/978-94-007-7584-8_7 . hal-01110207

\section{HAL Id: hal-01110207 \\ https://hal.science/hal-01110207}

Submitted on 27 Jan 2015

HAL is a multi-disciplinary open access archive for the deposit and dissemination of scientific research documents, whether they are published or not. The documents may come from teaching and research institutions in France or abroad, or from public or private research centers.
L'archive ouverte pluridisciplinaire HAL, est destinée au dépôt et à la diffusion de documents scientifiques de niveau recherche, publiés ou non, émanant des établissements d'enseignement et de recherche français ou étrangers, des laboratoires publics ou privés. 


\title{
Vector ordering and multispectral morphological image processing
}

\author{
Santiago Velasco-Forero and Jesus Angulo
}

\begin{abstract}
This chapter illustrates the suitability of recent multivariate ordering approaches to morphological analysis of colour and multispectral images working on their vector representation. On the one hand, supervised ordering renders machine learning notions and image processing techniques, through a learning stage to provide a total ordering in the colour/multispectral vector space. On the other hand, anomaly-based ordering, automatically detects spectral diversity over a majority background, allowing an adaptive processing of salient parts of a colour/multispectral image. These two multivariate ordering paradigms allow the definition of morphological operators for multivariate images, from algebraic dilation and erosion to more advanced techniques as morphological simplification, decomposition and segmentation. A number of applications are reviewed and implementation issues are discussed in detail.
\end{abstract}

\section{Introduction}

Problems on defining total order arise naturally in different aspects of science and engineering and their applications in daily life. In our context, "order" denotes an ordering principle: a pattern by which the elements of a given set may be arranged [13], and "total" means that the order is a binary relation antisymmetric, transitive and reflexive. Another name for a total order is linear order. It express the intuitive idea that you can picture a total order on a set $A$ as arranging the elements

Santiago Velasco-Forero

ITWM - Fraunhofer Institute, Kaiserslautern, Germany e-mail: velascoforero@itwm.fraunhofer.de

Jesus Angulo

CMM-Centre de Morphologie Mathématique, Mathématiques et Systèmes, MINES ParisTech; 35, rue Saint-Honoré, 77305 Fontainebleau CEDEX - Francee-mail: angulo@cmm.ensmp.fr 
of $A$ in a line. Not surprisingly, the task of defining a total order for a given set depends greatly on the prior knowledge provided. To illustrate this point, imagine a scenario where two researchers want to order a set of people. The first one defines minimum as the youngest person and the maximum as the oldest person, the second declares the minimum as the fattest one and the maximum as the thinnest one. Clearly, two persons that are similar according to the first researcher's setting might be dissimilar according to the second's. Accordingly, the complete list of ordered people can be totally different from one researcher to another. In the field of image processing, the definition of a total ordering among pixels of the image is the main ingredient of mathematical morphology techniques [20]. In this first part of this chapter, we study the case where "prior" knowledge about the spectral information of the background and the foreground on the image is available. We define a supervised ordering as a particular case of reduced ordering where the minimum (resp. maximum) value should be a pixel in the background (resp. foreground). This restriction can be included in the computation of the supervised ordering by using classical machine learning techniques, for instance, by support vector machines (SVM) [25]. Another possibility for known structure in the total ordering problem is to assume that the image is composed by two main components: background and foreground. Additionally, we include the assumption that the background is larger than the foreground. We uncover an interesting application of randomised approximation schemes in multivariate analysis [26]. To summarise, in this chapter a multispectral image is represented through a total ordering and it is analysed by mathematical morphology transformations. Prior information about the spectral information in the image is incorporated into the workflow of mathematical morphology transformations in two scenarios:

1. Spectral information about the background and the object of interest are available, i.e., "background/foreground training pixels".

2. Image can be considered as objects (foreground) over a majority background.

The remainder of this chapter is organised as follows. In Section 2, we present the fundamental definitions of mathematical morphology in a lattice formulation. The approach involving a preordering function is presented in Section 3. This section also contain examples specialising the general approach to more specific settings. Section 4 explains, implementation issues for any adjunction based morphological transformation. Finally, Section 5 concludes the chapter.

\section{Complete lattices and mathematical morphology}

\subsection{Mathematical morphology}

Basically, there are two points of view about mathematical morphological transformations: 1) connection based and 2) adjunction based. The first strategy deals with simplification of a given image in the partition space induced by its connected com- 


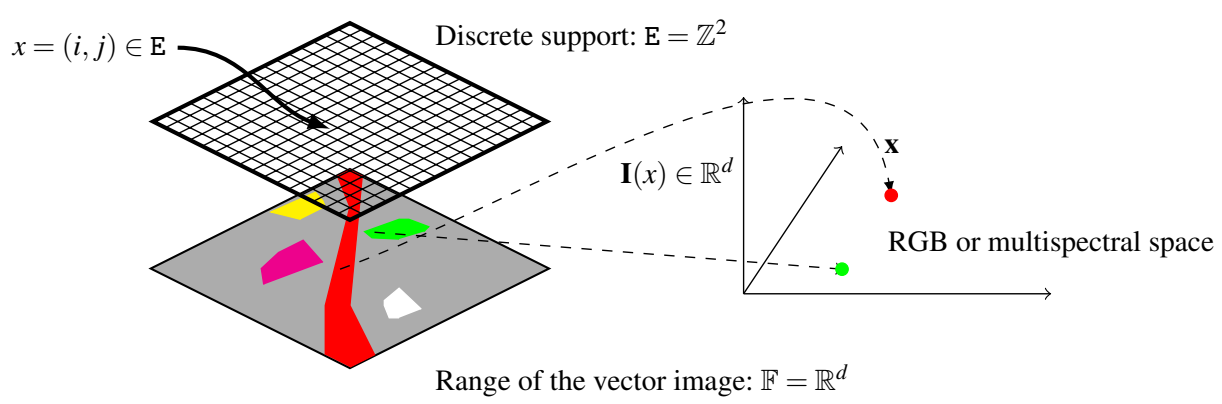

Fig. 1 Notation for a $d$-variate image, $\mathbf{I}: \mathrm{E} \rightarrow \mathbb{F}$. Note that the image I maps each spatial point $x$ to a vector $\mathbf{x}$ in three dimension for a RGB image or in dimension $d$ for the case of a multispectral image.

ponents $[17,18,21]$. The second perspective analyses an image by composition of two basic transformations, dilation and erosion, which form a Galois connection [9]. In this section we provide the theoretical background of mathematical morphology in its formulation based on adjunction, i.e. by using dilation/erosion operators. Our approach does not include the "connectivity approach". We refer keen readers to [21] for a comprehensive review of connective morphology.

\subsection{Fundamental definitions}

Let us introduce the notation for a multidimensional image, as it is illustrated in Fig. 1, where the object of interest is a $d$-dimensional image (denoted by $\mathbf{I}$ ) which maps the spatial support $\mathrm{E}$ to the vector support $\mathbb{F}$, i.e.,

$$
\begin{aligned}
\mathbf{I}: \quad \mathrm{E} & \rightarrow \mathbb{F}=\mathbb{R}^{d} \\
x & \rightarrow \mathbf{x}
\end{aligned}
$$

Given a vector image $\mathbf{I} \in \mathscr{F}(\mathrm{E}, \mathbb{F})$, i.e. is a mapping from the spatial support to the vector space of dimensions $d$. Theoretical formulation of mathematical morphology is nowadays phrased in terms of complete lattices and operators defined on them. For a detailed exposition on complete lattice theory in mathematical morphology, we refer to Chapter 2 (J. Serra and C. Ronse) in [16].

Definition 1 (Complete Lattice) A space $\mathscr{L}$ endowed with a partial order $\leq$ is called a complete lattice, denoted $(\mathscr{L}, \leq)$ if every subset $\mathscr{M} \subseteq \mathscr{L}$ has both supremum (join) $\bigvee \mathscr{M}$ and infimum (meet) $\wedge \mathscr{M}$.

A minimum (or least) $\perp \in \mathscr{M}$ is an element which is least than or equal to any other element of $\mathscr{M}$, that is, $r \in \mathscr{M} \Rightarrow \perp \leq r$. We denote the minimum of $\mathscr{L}$ by 
$\perp$. Equivalently, a maximum (largest) $\top$ in $\mathscr{M}$ is the greatest element of $\mathscr{M}$, that is, $r \in \mathscr{M} \Rightarrow r \leq \top$. We denote the maximum of $\mathscr{L}$ by $\top$.

Definition 2 (Dilation/Erosion) A mapping $f: \mathscr{L}_{1} \rightarrow \mathscr{L}_{2}$ of a complete lattice $\mathscr{L}_{1}$ into a complete lattice $\mathscr{L}_{2}$ is said to be a dilation if $f\left(\vee_{j \in J} r_{j}\right)=\vee_{j \in J} f\left(r_{j}\right)$ for all families $\left(r_{j}\right)_{j \in J}$ of elements in $\mathscr{L}_{1}$. A mapping is said to be an erosion if $f\left(\wedge_{j \in J} r_{j}\right)=$ $\wedge_{j \in J} f\left(r_{j}\right)$ for all families $\left(r_{j}\right)_{j \in J}$ of elements in $\mathscr{L}_{1}$.

The important relationship between dilation and erosion is that they are dual concepts from the lattice point of view. [9] showed that for any complete lattice $\mathscr{L}$, we always have a dual isomorphism between the complete lattice of dilation on $\mathscr{L}$ and the complete lattice of erosions on $\mathscr{L}$. This dual isomorphism is called by Serra ([20], Chapter 1) the morphological duality. In fact it is linked to what one calls Galois connections in lattice theory, as we will see at the end of this section.

Definition 3 (Adjunction) Let $\delta, \varepsilon \in \mathscr{L} \rightarrow \mathscr{L}$. Then we say that $(\varepsilon, \delta)$ is an adjunction of every $r, s \in \mathscr{L}$, we have

$$
\delta(r) \leq s \Longleftrightarrow r \leq \varepsilon(s)
$$

In an adjunction $(\varepsilon, \delta), \varepsilon$ is called the upper adjoint and $\delta$ the lower adjoint.

Proposition 1 ([9] p. 264) Let $\delta, \varepsilon \in \mathscr{L} \rightarrow \mathscr{L}$. If $(\varepsilon, \delta)$ is an adjunction, then $\delta$ is a dilation and $\varepsilon$ is an erosion.

Definition 4 (Galois connection) Let $\mathscr{L}_{1}$ and $\mathscr{L}_{2}$ be lattices and let $\alpha: \mathscr{L}_{1} \rightarrow \mathscr{L}_{2}$ and $\beta: \mathscr{L}_{2} \rightarrow \mathscr{L} 1$ satisfy the following conditions.

1. For $r, s \in \mathscr{L}_{1}$, if $r \leq s$, then $\alpha(s) \leq \alpha(r)$.

2. For $r, s \in \mathscr{L}_{1}$, if $r \leq s$, then $\beta(s) \leq \beta(r)$.

3. For $r \in \mathscr{L}_{1}, \beta \alpha(r) \leq r$.

4. For $r \in \mathscr{L}_{2}, \alpha \beta(r) \leq r$.

Then $(\alpha, \beta)$ is a Galois connection between $\mathscr{L}_{1}$ and $\mathscr{L}_{2}$.

Proposition 2 Let the lattices $\mathscr{L}_{1}$ and $\mathscr{L}_{2}$, maps $\alpha: \mathscr{L}_{1} \rightarrow \mathscr{L}_{2}$ and $\beta: \mathscr{L}_{2} \rightarrow \mathscr{L}_{1}$ a Galois connection. Then the following condition holds for all $r \in \mathscr{L}_{1}$ and $s \in \mathscr{L}_{2}$ :

$$
s \leq \alpha(r) \Longleftrightarrow r \leq \beta(s)
$$

Clearly an adjunction in $\mathscr{L}$ is a Galois connection between the dual $(\mathscr{L}, \geq)$ and $(\mathscr{L}, \leq)$ (indeed, compare definition 3 and proposition 2).

At this point, we can see that definition of erosion/dilation on a image requires a complete lattice structure, i.e., a total ordering ${ }^{1}$ among the pixels to be analysed. However, there is not difficult to see that the idea of order is entirely absent from multivariate scene, i.e., there is no unambiguous means of defining the minimum

${ }^{1}$ Theoretically, a partial ordering is enough but to make easier the presentation we analyse the case of total ordering. 
and maximum values between two vectors of more than one dimension. Accordingly, the extension of mathematical morphology to vector spaces, for instance, colour/multi/hyper/ultraspectral images, is neither direct nor trivial because the pixels in the images are vectors. We refer keen readers to [1][2] for a comprehensive review of vector morphology.

\subsection{Preorder by h-function}

Let $\mathrm{E}$ be a nonempty set and assume that $\mathscr{L}$ is a complete lattice. Let $h: \mathrm{E} \rightarrow \mathscr{L}$ be a surjective mapping. Define an equivalence relation $=_{h}$ on E as follows: $x={ }_{h} y \Leftrightarrow$ $h(x)=h(y) \quad \forall x, y \in$ E. As it was defined in [8], we refer by $\leq_{h}$ the $h$-ordering given by the following relation on $\mathrm{E}$

$$
\forall x, y \in \mathrm{E}, \quad x \leq_{h} y \Leftrightarrow h(x) \leq h(y)
$$

Note that $\leq_{h}$ preserves reflexivity $\left(x \leq_{h} x\right)$ and transitivity $\left(x_{1} \leq_{h} x_{2}\right.$ and $x_{2} \leq_{h} x_{3} \Rightarrow$ $\left.x_{1} \leq_{h} x_{3}\right)$. However, $\leq_{h}$ is not a partial ordering because $x \leq_{h} y$ and $y \leq_{h} x$ implies only that $x={ }_{h} y$ but not $x=y$. Note that $h$-ordering is a preorder in E.

An operator $\psi: \mathrm{E} \rightarrow \mathrm{E}$ is $h$-increasing if $x \leq_{h} y$ implies that $\psi(x) \leq_{h} \psi(y)$. Additionally, since $h$ is surjective, an equivalence class is defined by $\mathscr{L}[r]=\{y \in$ $\mathrm{E} \mid h(y)=r\}$. The Axiom of Choice [8] implies that there exist mappings $h^{\leftarrow}: \mathscr{L} \rightarrow \mathrm{E}$ such that $h h^{\leftarrow}(r)=r$, for $r \in \mathscr{L}$. Unless $h$ is injective, there exist more than one such $h^{\leftarrow}$ mappings: $h^{\leftarrow}$ is called the semi-inverse of $h$. Note that $h^{\leftarrow} h$ is not the identity mapping in general (but $h^{\leftarrow} h={ }_{h} \mathrm{id}$ ). However, we have that for any $h$-increasing $\psi: \mathrm{E} \rightarrow \mathrm{E}$ the result $\psi h^{\leftarrow} h={ }_{h} \psi$ and hence $h \psi h^{\leftarrow} h=h \psi$. Let us introduce $\widetilde{\psi}$ the operator associated to $\psi$ in the lattice $\mathscr{L}$. A mapping $\psi: \mathrm{E} \rightarrow \mathrm{E}$ is $h$-increasing if and only if there exists an increasing mapping $\widetilde{\psi}: \mathscr{L} \rightarrow \mathscr{L}$ such that $\tilde{\psi} h=h \psi$. The mapping $\widetilde{\psi}$ is uniquely determined by $\psi$ and can be computed from

$$
\widetilde{\psi}=h \psi h^{\leftarrow}
$$

We can now define the $h$-erosion and $h$-dilation. Let $\varepsilon, \delta: \mathrm{E} \rightarrow \mathrm{E}$ be two mappings with the property

$$
\delta(x) \leq_{h} y \Leftrightarrow x \leq_{h} \varepsilon(y), \quad \forall x, y \in \mathrm{E}
$$

then the pair $(\varepsilon, \delta)$ is called an $h$-adjunction. Moreover, let $(\varepsilon, \delta)$ be $h$-increasing mappings on E, and let $\varepsilon \mapsto^{h} \widetilde{\varepsilon}, \delta \mapsto^{h} \widetilde{\delta}$. Then $(\varepsilon, \delta)$ is an $h$-adjunction on $\mathrm{E}$ if and only if $(\widetilde{\varepsilon}, \widetilde{\delta})$ is an adjunction on the lattice $\mathscr{L}$. Therefore a mapping $\delta$ (resp. $\varepsilon$ ) on $\mathrm{E}$ is called $h$-dilation (resp. $h$-erosion) if $\widetilde{\delta}$ (resp. $\widetilde{\varepsilon}$ ) is a dilation (resp. erosion) on $\mathscr{L} . h$-adjunctions inherit a large number of properties from ordinary adjunctions between complete lattices. Assume that $(\varepsilon, \delta)$ is an $h$-adjunction then

$$
\gamma=\delta \varepsilon \leq_{h} \mathrm{id} \leq_{h} \varphi=\varepsilon \delta
$$


Hence, $\gamma$ is $h$-anti-extensive and $\phi$ is $h$-extensive. The operator $\gamma$ on E is called $h$-opening if the operator $\widetilde{\gamma}$ on $\mathscr{L}$ determined by $\gamma \mapsto{ }^{h} \widetilde{\gamma}$ is an opening. The operator $\gamma$ is also $h$-increasing and satisfies $\gamma \gamma={ }_{h} \gamma$ (h-idempotency). The $h$-closing is similarly defined.

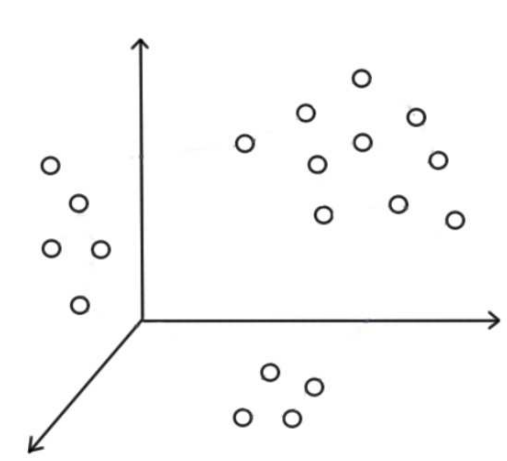

(a) Spectral information in $\mathbb{R}^{d}$.

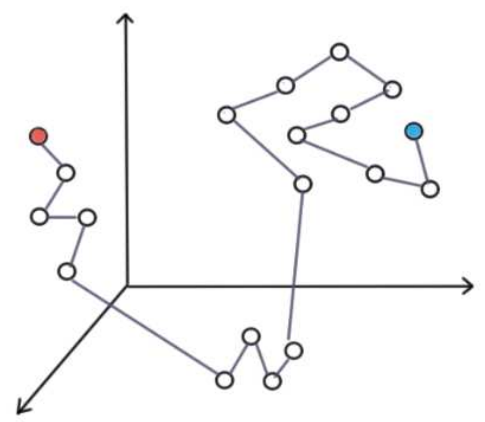

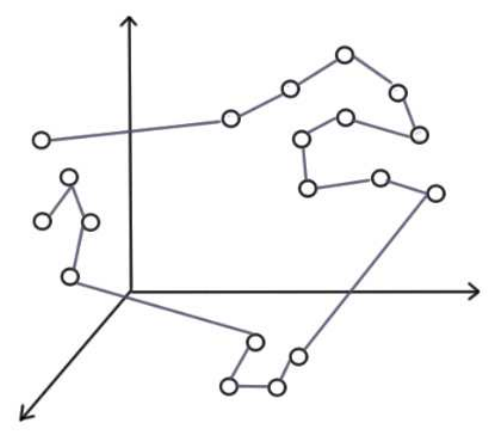

(b) Example of linear ordering on (a)

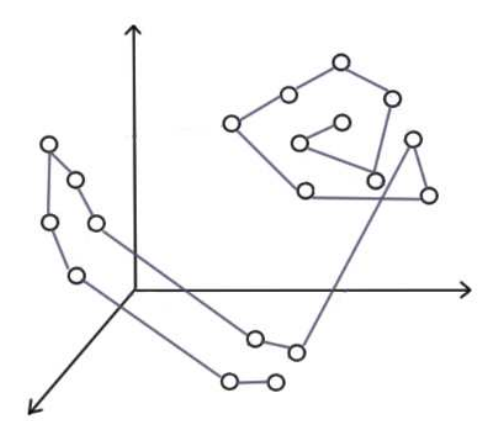

(c) Supervised ordering, the set of pixels are (d) Anomaly based ordering, the set of pixanalysed through a total order relation. els are analysed through a total order relation.

Fig. 2 In the complete lattice representation, the set of pixels are analysed through a linear order relation denoted by $h: \mathbb{R}^{d} \rightarrow \mathscr{L}$ in their spectral representation. In (c) the linear order starts from the background pixel (in red) and ends at the foreground pixel (in blue). In (d) the linear order starts from the centre of the greatest cluster in $\mathbb{R}^{d}$. 


\subsection{Morphological analysis on the h-function}

From the preliminary section we have the ingredients to define morphological colour $\left(\mathbb{F}=\mathbb{R}^{3}\right)$ and multispectral $\left(\mathbb{F}=\mathbb{R}^{d}\right)$ erosion and dilation. We limit here our developments to the flat operators, i.e., the structuring elements are planar shapes. The nonplanar structuring functions are defined by weighting values on their support [19]. Let us assume that we have an adaptive mapping ${ }^{2} h: \mathbb{R}^{d} \rightarrow \mathbb{R}$. The $h$-erosion $\varepsilon_{\mathrm{SE}, h}(\mathbf{I})$ and $h$-dilation $\delta_{\mathrm{SE}, h}(\mathbf{I})$ of an image $\mathbf{I}$ at pixel $x \in \mathrm{E}$ by the structuring element $\mathrm{SE} \subset \mathrm{E}$ are the two mappings $\mathscr{F}(\mathrm{E}, \mathbb{F}) \rightarrow \mathscr{F}(\mathrm{E}, \mathbb{F})$ defined respectively by

$$
h\left(\varepsilon_{\mathrm{SE}, h}(\mathbf{I})(x)\right)=\widetilde{\varepsilon}_{\mathrm{SE}}(h(\mathbf{I}))(x),
$$

and

$$
h\left(\delta_{\mathrm{SE}, h}(\mathbf{I})(x)\right)=\widetilde{\delta}_{\mathrm{SE}}(h(\mathbf{I}))(x),
$$

where $\widetilde{\varepsilon}_{\mathrm{SE}}(I)$ and $\widetilde{\delta}_{\mathrm{SE}}(I)$ are the standard numerical flat erosion and dilation of image $I \in \mathscr{F}(\mathrm{E}, \mathscr{L})$ :

$$
\begin{aligned}
& \widetilde{\varepsilon}_{\mathrm{SE}}(I)(x)=\left\{I(y): I(y)=\bigwedge[I(z)], z \in \mathrm{SE}_{x}\right\} \\
& \widetilde{\delta}_{\mathrm{SE}}(I)(x)=\left\{I(y): I(y)=\bigvee[I(z)], z \in \check{\mathrm{SE}}_{x}\right\}
\end{aligned}
$$

with $\mathrm{SE}_{x}$ being the structuring element centred at point $x$ and $\breve{\mathrm{SE}}$ is the reflected structuring element. If the inverse mapping $h^{-1}$ is defined, the $h$ - erosion and dilation can be explicitly written as:

$$
\varepsilon_{\mathrm{SE}, h}(\mathbf{I})(x)=h^{-1}\left(\widetilde{\varepsilon}_{\mathrm{SE}}(h(\mathbf{I}))\right)(x)
$$

and

$$
\delta_{\mathrm{SE}, h}(\mathbf{I})(x)=h^{-1}\left(\widetilde{\delta}_{\mathrm{SE}}(h(\mathbf{I}))\right)(x) .
$$

Of course, the inverse $h^{-1}$ only exists if $h$ is injective. In practice, we can impose the invertibility of $h$ by considering a lexicographic ordering for equivalence class $\mathscr{L}[\mathbf{x}]$. In fact, this solution involves a structure of total ordering which allows to compute directly the $h$-erosion and dilation without using the inverse mapping, i.e.,

$$
\varepsilon_{\mathrm{SE}, h}(\mathbf{I})(x)=\left\{\mathbf{I}(y): \mathbf{I}(y)=\bigwedge_{h}[\mathbf{I}(z)], z \in \mathrm{SE}_{x}\right\}
$$

and

$$
\delta_{\mathrm{SE}, h}(\mathbf{I})(x)=\left\{\mathbf{I}(y): \mathbf{I}(y)=\bigvee_{h}[\mathbf{I}(z)], z \in \check{\mathrm{SE}}_{x}\right\}
$$

\footnotetext{
${ }^{2}$ Adaptive in the sense that the mapping depend on the information contained in a multivariate image I. The correct notation should be $h(\cdot ; \mathbf{I})$. However, in order to make easier the understanding of the section we use $h$ for adaptive mapping.
} 
where $\Lambda_{h}$ and $\bigvee_{h}$ are respectively the infimum and supremum according to the ordering $\leq_{h}$. Starting from the $h$-adjunction $\left(\varepsilon_{\mathrm{SE}, h}(\mathbf{I}), \delta_{\mathrm{SE}, h}(\mathbf{I})\right)$, all the morphological filters such as the opening and closing have their $h$-counterpart, e.g., the $h$ opening and closing are defined as

$$
\gamma_{\mathrm{SE}, h}(\mathbf{I})=\delta_{\mathrm{SE}, h}\left(\varepsilon_{\mathrm{SE}, h}(\mathbf{I})\right), \quad \varphi_{\mathrm{SE}, h}(\mathbf{I})=\varepsilon_{\mathrm{SE}, h}\left(\delta_{\mathrm{SE}, h}(\mathbf{I})\right)
$$

Similarly, any other mathematical morphology operator based on adjunction operators can be also extended to multivariate images. For instance, geodesic operators as opening by reconstruction[22], levelings [14], additive morphological decompositions [27] and so on.

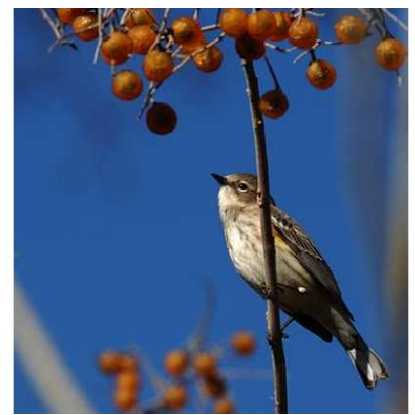

(a) Original Colour image denoted by $\mathbf{I}$

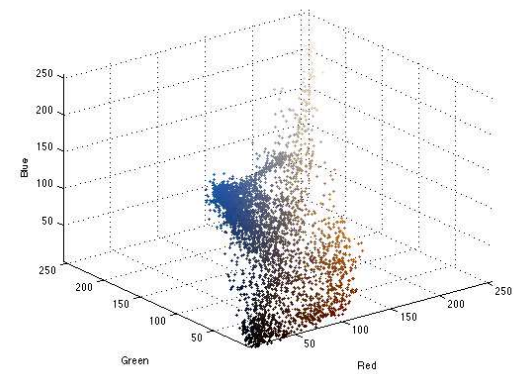

(b) Scatterplot of the three-channel image $\mathbf{X}_{\mathbf{I}}$.

Fig. 3 Spectral representation of a colour image in the RGB space. A spatial position $x$ in the image I contains three coordinates in the RGB-space represented by $\mathbf{x}$.

\section{Pre-ordering a vector space}

Let $\mathbf{X}_{\mathbf{I}}$ be the set of vector values of a given image $\mathbf{I}$, which can be viewed as a cloud of points in $\mathbb{F}$. Fig. 3 shows an example of colour image $\mathbf{I}$, and its spectral representation as points $\mathbf{X}_{\mathbf{I}}$. In general, pixel values in multispectral images are vectors defined in $\mathbb{F}=\mathbb{R}^{d}$. From previous section, for a given multivariate image $\mathbf{I}: \mathbf{E} \rightarrow \mathbb{R}^{d}$, the challenge to build complete lattice structures is to define a mapping $h: \mathbb{R}^{d} \rightarrow \mathscr{L}$, to obtain a mapping $\mathrm{E} \rightarrow \mathscr{L}$, where $\mathscr{L}$ is a lattice. In this chapter, we consider the lattice $\mathscr{L}$ of the extended real line $(\overline{\mathbb{R}}, \leq)$ using $\overline{\mathbb{R}}=\mathbb{R} \cup\{-\infty,+\infty\}$ and $\leq$ as the "less than or equal to" relation (the natural partial ordering). Many authors have already worked in this idea $[1,2,3,25]$. Basically, three family of reduced mappings $h$ for a given $\mathbf{x}=\left(\mathrm{x}_{1}, \mathrm{x}_{2}, \ldots, \mathrm{x}_{d}\right) \in \mathbb{R}^{d}$ can be defined as it is illustrated in Table 1 . 


\begin{tabular}{|c|c|}
\hline Type of reduced mapping & $h(\mathbf{x} ; \cdot)$ \\
\hline \multicolumn{2}{|c|}{ Unsupervised } \\
\hline Linear dimensionality reduction[10] & $h_{\mathrm{PCA}}(\mathbf{x})=\sum_{i=1}^{d} \lambda^{i} \mathbf{x}_{i}$ \\
\hline Local unsupervised [1] & $h_{\mathrm{LPCA}}(\mathbf{x})=\sum_{i=1}^{d} \lambda_{\mathbf{x}}^{i} \times_{i}$ \\
\hline \multicolumn{2}{|c|}{ Distance based } \\
\hline Referenced ordering [1] & $h_{\mathrm{REF}}(\mathbf{x} ; T)=\sum_{i=1}^{|T|} \lambda_{\mathbf{x}}^{i} \mathbf{K}\left(\mathbf{t}_{i}, \mathbf{x}\right)$ \\
\hline Supervised [25] & $h_{\mathrm{SUPER}}(\mathbf{x} ; B, F)=\sum_{k=1}^{B \mid} \lambda_{\mathbf{x}}^{k} \mathbf{K}\left(\mathbf{b}_{k}, \mathbf{x}\right)+\sum_{j=1}^{|F|} \lambda_{\mathbf{x}}^{j} \mathbf{K}\left(\mathbf{f}_{j}, \mathbf{x}\right)$ \\
\hline \multicolumn{2}{|c|}{ Anomaly based } \\
\hline Projection Depth [26] & $h_{\mathrm{ANOM}}(\mathbf{x} ; \mathbf{I})=\max _{\mid \mathbf{u} \|=1} \frac{\left|\mathbf{u}^{T} \mathbf{x}-\operatorname{med}\left(\mathbf{u}^{T} \mathbf{X}_{\mathbf{I}}\right)\right|}{\operatorname{mad}\left(\mathbf{u}^{T} \mathbf{X}_{\mathbf{I}}\right)}$ \\
\hline
\end{tabular}

Table 1 Different adaptive multivariate orderings implemented by $h$-mapping based reduced ordering. Note that $\mathbf{x}$ is a vector which $d$ components, $\mathrm{x}_{1}, \mathrm{x}_{2}, \ldots, \mathrm{x}_{d}, \mathbf{K}: \mathbb{R}^{d} \times \mathbb{R}^{d} \rightarrow \mathbb{R}^{+}$is a kernelinduced distance, the sets $B=\left\{\mathbf{b}_{1}, \mathbf{b}_{2}, \ldots, \mathbf{b}_{|B|}\right\}, F=\left\{\mathbf{f}_{1}, \mathbf{f}_{2}, \ldots, \mathbf{f}_{|F|}\right\}$ and $T=\left\{\mathbf{t}_{1}, \mathbf{t}_{2}, \ldots, \mathbf{t}_{|T|}\right\}$ are the background, foreground and training, respectively. The matrix $\mathbf{X}_{\mathbf{I}}$ is an array containing the pixel information of a multidimensional image denoted by $\mathbf{I}$, i.e., $\mathbf{X}_{\mathbf{I}}=\left[\mathbf{x}_{1} \mathbf{x}_{2} \ldots \mathbf{x}_{n}\right]$ where $\mathbf{X}_{\mathbf{I}}$ has $d$ rows and $n$ columns. The orders considered here depend on an input image I. A schematic representation of these orders is given in Fig.2.

\subsection{Unsupervised ordering}

That can be obtained by using the more representative projection in a statistical dimensional reduction technique, for example a linear approach as PCA [10] or some non-linear projections approach [12]. To illustrate, we consider the first projection to induce the ordering, i.e., $\mathbf{x}_{1} \leq \mathbf{x}_{2} \Longleftrightarrow h_{\mathrm{PCA}}(\mathbf{x}) \leq h_{\mathrm{PCA}}\left(\mathbf{x}_{2}\right)$, where $h_{\mathrm{PCA}}$ is the first eigenvector of the centred covariance matrix $\mathbf{X}_{\mathbf{I}}^{T} \mathbf{X}_{\mathbf{I}}$. The intuition behind this approach is simple and clear: pixels are ordered according to their representation in the projection with greatest variance. An example is illustrated in Fig. 4(b). In this example, we can see that the induced minimum and maximum have no practical interpretation. A second disadvantage is that in this case, the minimum or maximum can drastically change by altering "a pixel" or a "limited number of pixels" in the original image $\mathbf{I}$.

\subsection{Distance based ordering}

Let us focus on the case of $h$-ordering based on distances. This approach is motivated by the intuition that order computation should be adaptive to prior information given by application interests.

\subsubsection{Referenced ordering}

As a starting point for distance based ordering, we consider the work of Angulo [1], who defines a function $h_{\mathrm{REF}}(\cdot, \mathbf{t})$ that computes the similarity for a given pixel 


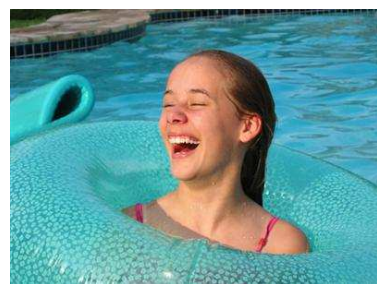

(a) Colour image: I

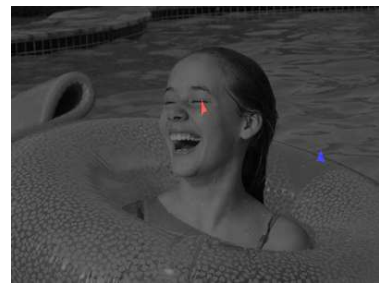

(d) $B$ and $F$ sets.

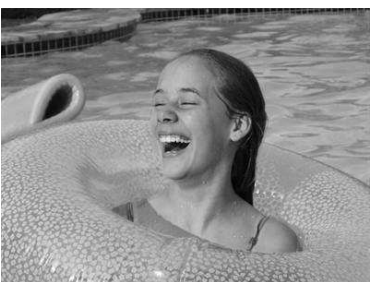

(b) $h_{\mathrm{PCA}}$

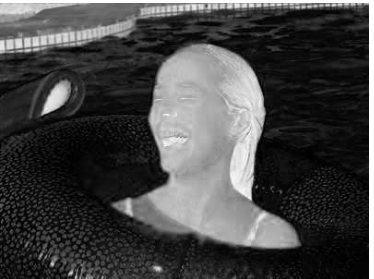

(e) $h_{\text {REF }}$

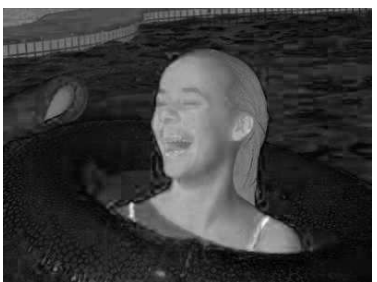

(c) $h_{\mathrm{ANOM}}$

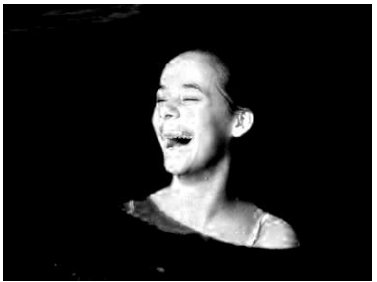

(f) $h_{\text {SUPER }}$

Fig. 4 Comparison of different $h$-mappings considered in this chapter for a given colour image. Referenced and supervised $h$-mappings requires prior information given by the sets $B$ and $F$. Anomaly based ordering is intrinsically adapted to the image.

$\mathbf{x}$ to a colour reference $\mathbf{t}$ by measuring its spectral distance, i.e., $\mathbf{x}_{1} \leq_{h_{\mathrm{REF}}} \mathbf{x}_{2} \Longleftrightarrow$ $\mathbf{K}\left(\mathbf{x}_{1}, \mathbf{t}\right) \leq \mathbf{K}\left(\mathbf{x}_{2}, \mathbf{t}\right)$, where $\mathbf{K}: \mathbb{R}^{d} \times \mathbb{R}^{d} \rightarrow \mathbb{R}^{+}$is a kernel-induced distance[15]. The original formulation in [1] uses the case of Euclidean distance in the colour space as kernel-induced distance ${ }^{3}$. Thus, the ordering based on a reference spectrum exhibits a lattice where the minimum has been fixed. However, that maximum is associated with the "farthest" vector but that does not have a simple interpretation. To illustrate the result of this approach, we generalise the definition of a referenced order for a training set $T$ as follows, $\mathbf{x}_{1} \leq_{h_{\mathrm{REF}}} \mathbf{x}_{2} \Longleftrightarrow \min _{i}\left\|\mathbf{x}_{1}-\mathbf{t}_{i}\right\| \geq \min _{i}\left\|\mathbf{x}_{2}-\mathbf{t}_{i}\right\|$ for all $\mathbf{t}_{i} \in T$. The geometric interpretation is that $h_{\mathrm{REF}}(\mathbf{x} ; T)$ is basically the distance in $L_{\infty}$ of $\mathbf{x}$ to the convex hull of vectors in $T$ (if $\mathbf{x}$ is not in the convex hull). Thus, is not so difficult to see that $h_{\mathrm{REF}}$ can be expressed as $h_{\mathrm{REF}}(\mathbf{x} ; T)=\sum_{i=1}^{|T|} \lambda_{\mathbf{x}}^{i} \mathbf{K}\left(\mathbf{t}_{i}, \mathbf{x}\right)$ where $\lambda_{\mathbf{x}}^{i} \neq 0$ only for $\arg \min _{i}\left\|\mathbf{x}-\mathbf{t}_{i}\right\|$. Fig. 4(e) shows the referenced mapping for the colour image in Fig. 4(a). The training set are the pixel in the red region of Fig 4(d). Note that $h_{\mathrm{REF}}$ "detects" the girl but at the same time the border of the swimming-pool. Associated morphological adjunction and gradient are illustrated in Fig. 5(g-i).

\footnotetext{
${ }^{3}$ In this case the sense of the inequality change, i.e., $\mathbf{x}_{1} \leq{ }_{h_{\mathrm{REF}}} \mathbf{x}_{2} \Longleftrightarrow\left\|\mathbf{x}_{1}-\mathbf{t}||^{2} \geq\right\| \mathbf{x}_{2}-\mathbf{t} \mid \|^{2}$.
} 


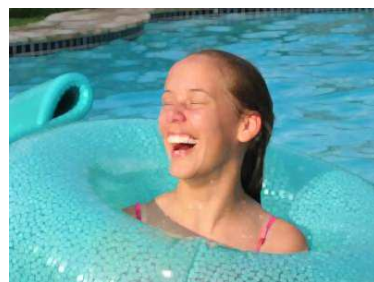

(a) $\delta_{\mathrm{SE}, h_{\mathrm{PCA}}}(\mathbf{I})$

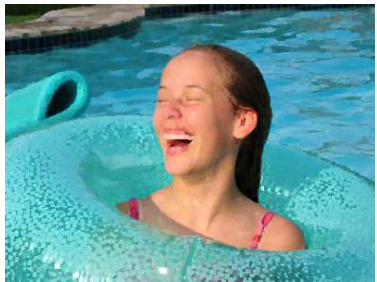

(d) $\delta_{\mathrm{SE}, h_{\mathrm{ANOM}}}(\mathbf{I})$

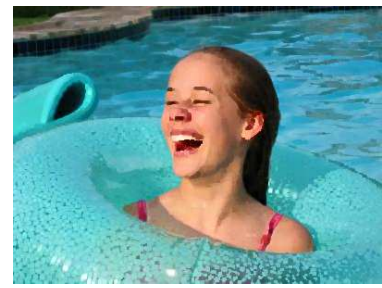

(g) $\delta_{\mathrm{SE}, h_{\mathrm{REF}}}(\mathbf{I})$

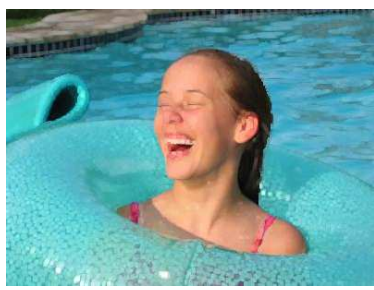

(j) $\delta_{\mathrm{SE}, h_{\mathrm{SUPER}}}(\mathbf{I})$

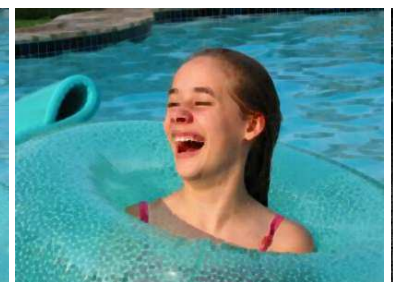

(b) $\varepsilon_{\mathrm{SE}, h_{\mathrm{PCA}}}(\mathbf{I})$

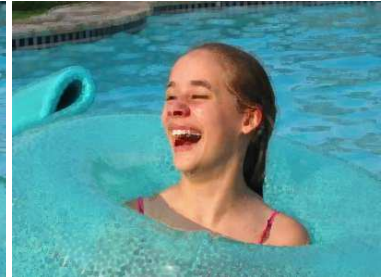

(e) $\varepsilon_{\mathrm{SE}, h_{\mathrm{ANOM}}}(\mathbf{I})$

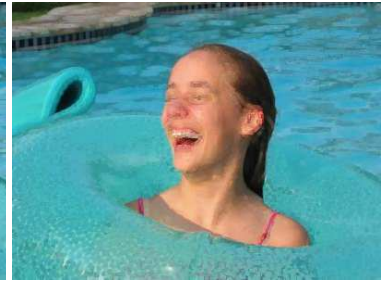

(h) $\varepsilon_{\mathrm{SE}, h_{\mathrm{REF}}}(\mathbf{I})$

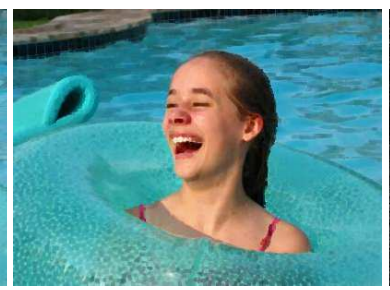

(k) $\varepsilon_{\mathrm{SE}, h_{\mathrm{SUPER}}}(\mathbf{I})$

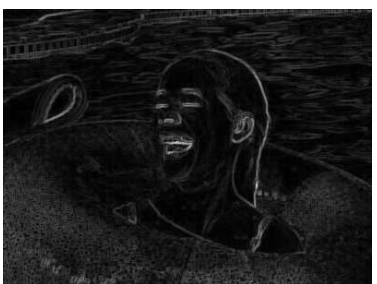

(c) Gradient by $h_{\mathrm{PCA}}$

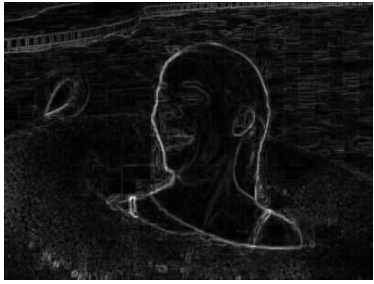

(f) Gradient by $h_{\text {ANOM }}$

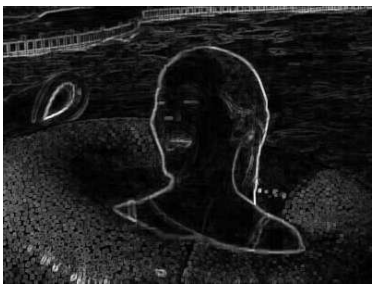

(i) Gradient by $h_{\mathrm{REF}}$

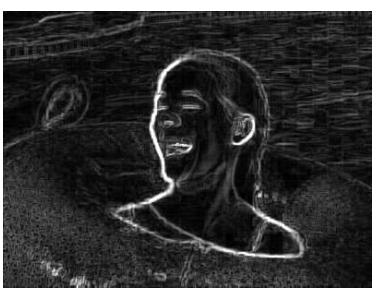

(1) Gradient by $h_{\text {SUPER }}$

Fig. 5 Comparison of colour dilation, erosion and associated gradient using different $h$-orderings (see Table 1). Gradients have been normalised from 0 to 1 to make easier the visual comparison.

\subsubsection{Supervised ordering}

A most general formulation for distance based ordering has been introduced in [25]. It defines a $h$-supervised ordering for every vector $\mathbf{x} \in \mathbb{R}^{d}$ based on the subsets $B=\left\{\mathbf{b}_{1}, \ldots, \mathbf{b}_{|B|}\right\}$ and $F=\left\{\mathbf{f}_{1}, \ldots, \mathbf{f}_{|F|}\right\}$, as a $h$-ordering that satisfies the following conditions: $h(\mathbf{b})=\perp$ then $\mathbf{b} \in \mathrm{B}$, and $h(\mathbf{f})=\top$ then $\mathbf{f} \in F$. Note that $\perp, \top$ are the smallest and largest element in the lattice $\mathscr{L}$. Such an $h$-supervised ordering is de- 
noted by $h_{\text {SUPER }}(\cdot ; B, F)$. Fig. 2(c) illustrates the main intuition for a $h$-supervised ordering function: it is a linear ordering from the pixels in background set $(\mathrm{B} \subseteq \mathscr{L}(\perp))$ to the ones in foreground set $(F \subseteq \mathscr{L}(\top))$. The main motivation of defining this new supervised ordering schema is to obtain maximum and minimum in the lattice $\mathscr{L}$ interpretable with respect to sets $\mathrm{B}$ and $F$. It is important to remind that max and min are the basic words in the construction of all mathematical morphology operators. At this point, the problem is how to define an adequate supervised ordering for a given vector space $\mathbb{F}$ and two pixel sets $B, F$. The approach introduced by [25] involves the computation of standard support vector machine (SVM) to solve a supervised classification problem to define the function $h_{\text {SUPER }}(\mathbf{x} ; B, F)$. An amusing geometrical interpretation is based on results from [4], in where the ordering induced by $h_{\text {SUPER }}$, corresponds to the signed distance to the separating plane between the convex hull associated to $F$ and the one containing the $B$. From [7], the solution of the classification case of SVM can be expressed as follows:

$$
h_{\mathrm{SUPER}}(\mathbf{x} ; B, F)=\sum_{k=1}^{|B|} \lambda^{k} \mathbf{K}\left(\mathbf{b}_{k}, \mathbf{x}\right)+\sum_{j=1}^{|F|} \lambda^{j} \mathbf{K}\left(\mathbf{f}_{j}, \mathbf{x}\right)
$$

where $\lambda^{k}$ are computed simultaneous as a quadratic programming optimisation problem [7]. For all the examples, given in this chapter we have used a Gaussian Kernel, with the Euclidean distance between colour or spectra, i.e. $\mathbf{K}\left(\mathbf{x}_{i}, \mathbf{x}_{j}\right)=$ $\exp \left(-c|| \mathbf{x}_{i}-\mathbf{x}_{j} \|^{2}\right)$, where the constant $c$ is obtained by cross-validation on the training set [7]. Results of this supervised ordering are illustrated in Fig. 4(f). The $h_{\text {SUPER }}$ matches our intuition of what should be maximum and what should be minimum in the image according to the couple $\{B, F\}$ in $4(\mathrm{~d})$. The supervised adjunction is shown in Fig. 5(j-k). Note that the supervised gradient in Fig.5(l) is better defined on the contour of the girl in comparison to unsupervised and referenced orders. A second example is presented fro the RGB image in Fig. 3 considering the training sets in Fig. 6(a). Note that the supervised lattice in Fig. 6(c), is a mapping from the spectral information to a linear ordering (from top-left corner to bottom right corner). One advantage of the definition of $h$-ordering on vector space is that it can be applied directly to multispectral or even hyperspectral images. In order to illustrate this flexibility, we present the case of a RGB and Near-infrared (NIR) image in Fig. 7(a-b) from [6]. The spectral information is considered on $\mathbb{R}^{4}$ and background and foreground sets are the spectra information contained in the marked regions in Fig. 7(c). For purposes such as segmentation, we would use inner/outer markersdriven watershed transformation [5]. Fig. 7(i) depicts the results of the segmentation from the same set of markers Fig. 7(c) in both orders: referenced (f) and supervised (i). Notice that, supervised approach matches better the general structure of the original multispectral image than referenced one. 


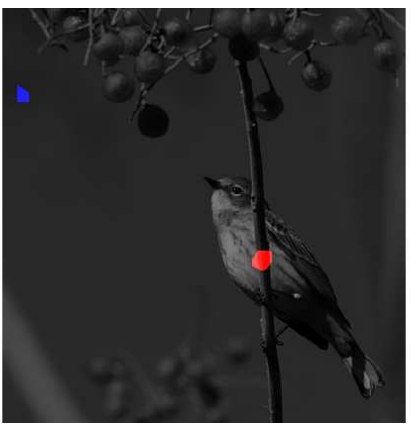

(a) Background/foreground training set

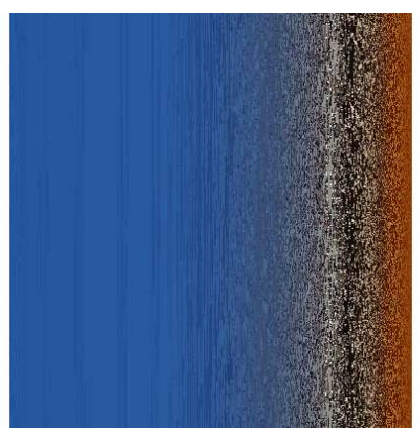

(c) Learned Order from (b).

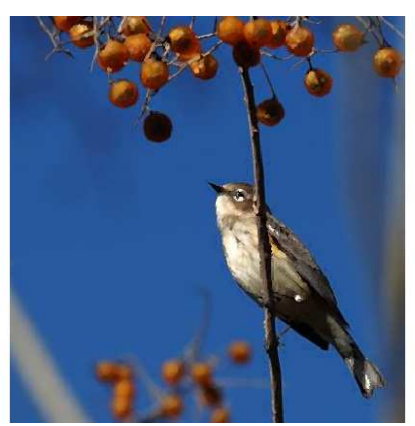

(e) $\varepsilon_{\mathrm{SE}, h_{\mathrm{SUPR}}}(\mathbf{I})$

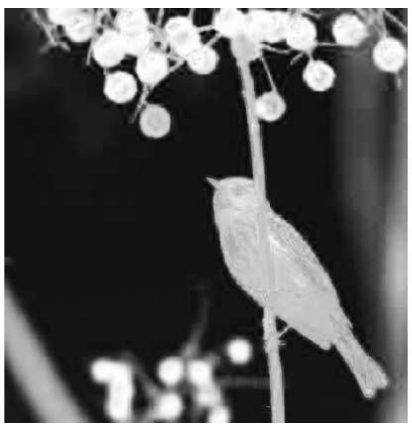

(b) $h_{\mathrm{SUPER}}(\cdot ; B, F)$

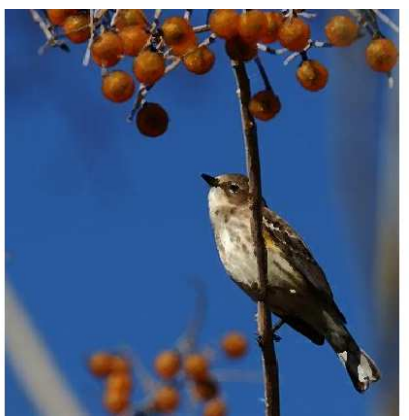

(d) $\delta_{\mathrm{SE}, h_{\mathrm{SUPER}}}(\mathbf{I})$

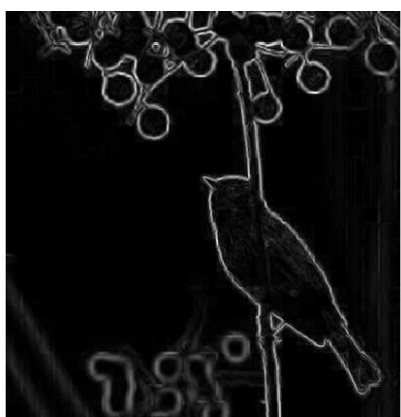

(f) Gradient by $h_{\text {SUPER }}$ in (b)

Fig. 6 Background pixels are in blue, and foreground ones in red. The minimum in the supervised ordering is placed at the top left corner and the maximum at the bottom right corner. Morphological operators are computed by using a square of side 3 pixels as $\mathrm{SE}$. 


\subsection{Ordering based on anomalies}

Distance based ordering approaches discussed above are valid if the pair set $(B, F)$ is available. Obviously, one cannot realistically believe that for every application the exact spectral information about the background of the image is available. Thus, if one gives up this paradigm, no other option different to unsupervised ordering remains. Therefore, in order to take advantage of the physical structure of an image, it was introduced in [26] an ordering based on "anomalies" with respect to a background associated to a majority of points. It is called depth ordering and is maximal in the "centre" of the spectral representation of a image $\mathbf{I}$ and it produces a vector ordering "centre-outward" to the outliers in the vector space $\mathbb{R}^{d}$. In this paradigm, the assumption of existence of an intrinsic background/foreground representation is required, i.e., given a vector image $\mathbf{I}: \mathrm{E} \rightarrow \mathbb{R}^{d}, \mathbf{X}_{\mathbf{I}}$ has can be decomposed as $\mathbf{X}_{\mathbf{I}}=\left\{\mathbf{X}_{B(\mathbf{I})}, \mathbf{X}_{F(\mathbf{I})}\right\}$ such that $\mathbf{X}_{B(\mathbf{I})} \cap \mathbf{X}_{F(\mathbf{I})}=\emptyset$ and $\operatorname{card}\left\{\mathbf{X}_{B(\mathbf{I})}\right\}>\operatorname{card}\left\{\mathbf{X}_{F(\mathbf{I})}\right\}$. Roughly speaking, the assumption means: (1) the image has two main components: the background and the foreground; (2) There are more pixels in the background than in the foreground. Several examples of these kind of functionals have been analysed in [24]. However, we limited ourselves to the statistical projection depth case presented in [26] and defined by

$$
h_{\mathrm{ANOM}}(\mathbf{x} ; \mathbf{I})=\sup _{\|\mathbf{u}\|=1} \frac{\left|\mathbf{u}^{T} \mathbf{x}-\operatorname{med}\left(\mathbf{u}^{T} \mathbf{X}_{\mathbf{I}}\right)\right|}{\operatorname{mad}\left(\mathbf{u}^{T} \mathbf{X}_{\mathbf{I}}\right)}
$$

where med denoted the univariate median and mad the median absolute deviation, i.e., the median of the differences with respect to the median. Not the that the superscript ${ }^{T}$ denotes matrix transposition. Let us now point out some aspects of (11) in order to better characterise it. First, it is a anomaly based ordering, due to the fact that if $\mathbf{X}_{\mathbf{I}} \sim \mathbb{N}(\mu, \Sigma)$ a Gaussian distribution with mean vector $\mu$ and covariance matrix $\Sigma$ then $h_{\text {ANOM }}(\mathbf{x} ; \mathbf{I})^{2} \propto(\mathbf{x}-\mu)^{T} \Sigma^{-1}(\mathbf{x}-\mu)$, the Mahalanobis distance (see [26] to details). Secondly, (11) is invariant to affine transformations in the vector space $\mathbb{R}^{d}$. Third, unfortunately, the exact computation of (11) is computationally intensive except when the number of pixels $n$ is very small. However, we can compute a stochastic approximation by using a large number of random projections $\mathbf{u}$ and computing the maximum for a given $\mathbf{x}$ [26].

To summarise the above, the statistical projection depth function in (11) induces an anomaly based ordering for images with background/foreground representation. That is an ordering based on a data-adapted function and in such a way that the interpretation of supremum and infimum operations is known a priori, because max values can be associated with "outlier" pixels in the high-dimensional space and min are "central" pixels in $\mathbb{R}^{d}$ space. A simple example is illustrated in Fig. 4(c) where (11) "detects" the girl thanks to the fact that her spectral information is unusual in comparison to the one from the swimming pool. 


\section{Implementation}

Once a $h$-ordering has been defined, it becomes easy in practice to implement morphological transformations on multidimensional images such as colour or multispectral ones. Actually, we can use a scalar to code each pixel on the image, and the standard morphological transformations for grayscale images can be used directly. The result is deciphered by mapping back the total ordering in to the vector space. An effective implementation using a look-up table has been presented in [23]. A pseudo-code for a multivariate erosion ${ }^{4}$ is shown in Algorithm 1 in Matlab notation. The index image and the sorted vector look-up table constructed above are used to generate an ordered table. At this point, any morphological transformation can be performed on the lattice image, which can be considered as a grey scale image. The output of the morphological transformation is converted back to the original vector space by replacing each pixel by its corresponding vector using a look-up table.

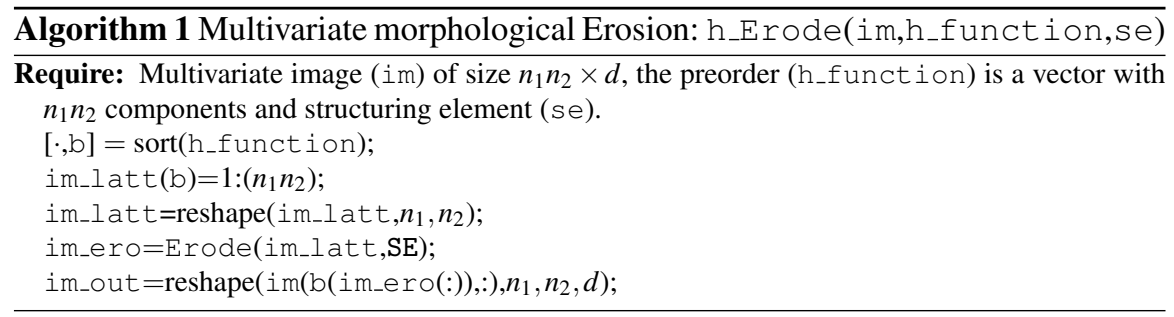

\section{Conclusions}

Mathematical morphology is a non-linear methodology for image processing based on a pair of adjoint and dual operators, dilation and erosion, used to compute sup/infconvolutions in local neighbourhoods. The extension of morphological operators to colour images has been the object of many works in the past; however, the generalisation of such colour approaches to multispectral images is not straightforward. In this chapter, we illustrated how kernel-based learning techniques and multivariate statistics can be exploited to design vector ordering and to include results of morphological operators in the pipeline of colour and multispectral image analysis. Two main families of ordering have been described. Firstly, we focused on the notion of supervised vector ordering which is based on a supervised learning formulation. A training set for the background and another training set for the foreground are needed as well as a supervised method to construct the ordering mapping. Secondly,

\footnotetext{
${ }^{4}$ It is important to note that any adjunction based morphological transformations as openings, closings, levelings and so on, can be implemented in similar way, i.e., by changing the function Erode by another grey scale morphological transformation.
} 


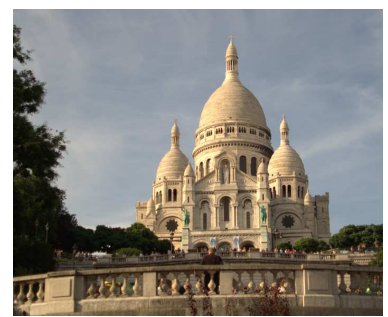

(a) Original image and training sets

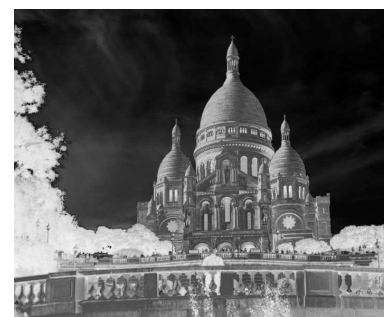

(d) $h_{\mathrm{REF}}(\cdot ; F)$

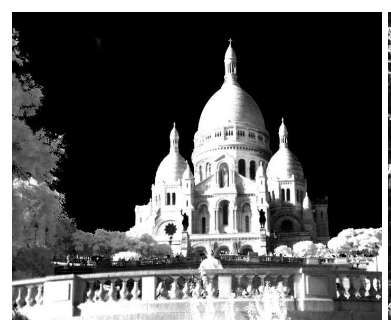

(g) $h_{\text {SUPER }}(\cdot ; B, F)$

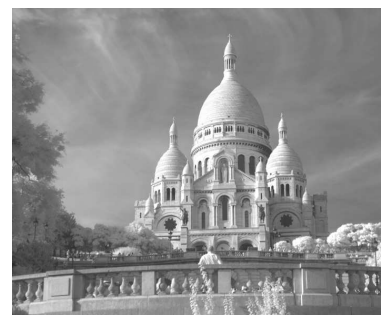

(b) NIR Channel

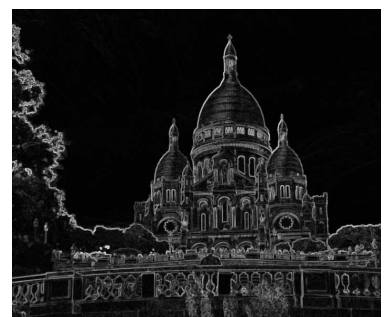

(e) Gradient of $h_{\mathrm{REF}}$

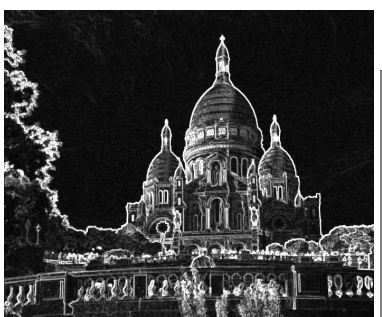

(h) Gradient of $h_{\text {SUPER }}$

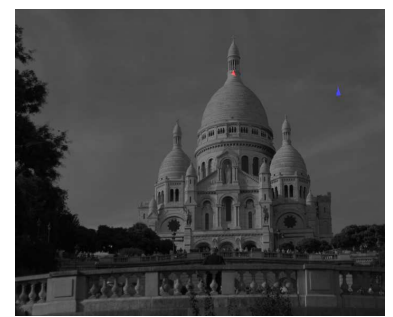

(c) Training sets $(F, B)$

(f) Marked watershed on $h_{\mathrm{REF}}$

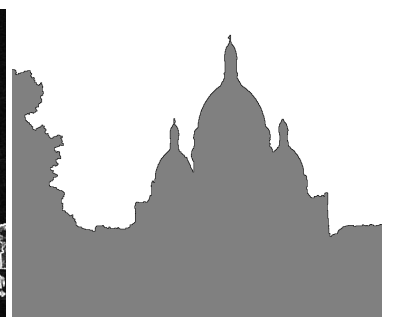

(i) Marked watershed on $h_{\text {SUPER }}$

Fig. 7 Effect of the inclusion of supervised ordering in marked based segmentation. The spectra information of RGB+NIR image (a) are considered as vectors in $\mathbb{R}^{4}$. Background (resp. foreground) set are blue (resp. red) pixels in (c).

we considered an (unsupervised) anomaly-based vector ordering based on statistical depth function computed by random projections. This leaded us to an intrinsic processing based on a background/foreground representation. We have illustrated in the examples the interest of morphological gradients (from pairs of multispectral dilation/erosion) for watershed segmentation. From a theoretical viewpoint, our framework is based on the theory of h-mapping adjunctions. 


\section{References}

1. J. Angulo. Morphological colour operators in totally ordered lattices based on distances: Application to image filtering, enhancement and analysis. Computer Vision and Image Understanding, 107(1-2):56-73, 2007.

2. E. Aptoula and S. Lefèvre. A comparative study on multivariate mathematical morphology. Pattern Recognition, 40(11):2914-2929, 2007.

3. V. Barnett. The ordering of multivariate data (with discussion). Journal of the Royal Stat. Soc. Series A, 139(3):318-354, 1976.

4. K. P. Bennett and E. J. Bredensteiner. Duality and geometry in svm classifiers. In In Proc. 17th International Conf. on Machine Learning, pages 57-64. Morgan Kaufmann, 2000.

5. S. Beucher and F. Meyer. The morphological approach to segmentation: the watershed transformation. Mathematical morphology in image processing. Optical Engineering, 34:433-481, 1993.

6. M. Brown and S. Süsstrunk. Multispectral SIFT for scene category recognition. In Computer Vision and Pattern Recognition (CVPR11), pages 177-184, Colorado Springs, June 2011.

7. N. Cristianini and J. Shawe-Taylor. An Introduction to support vector machines and other kernel based learning methods. Cambridge University Press, 2000.

8. J. Goutsias, H. J. A. M. Heijmans, and K.Sivakumar. Morphological operators for image sequences. Computer Vision and Image Understanding, 62(3):326-346, 1995.

9. H. J. A. M. Heijmans and C. Ronse. The algebraic basis of mathematical morphology. i. dilations and erosions. Comput. Vision Graph. Image Process., 50:245-295, June 1990.

10. I. T. Jolliffe. Principal Component Analysis. Springer-Verlag, 1986.

11. N. Kambhatla and T.K. Leen. Dimension reduction by local principal component analysis. Neural Comp., 9(7):1493-1516, 1997.

12. O. Lezoray, C. Charrier, and A. Elmoataz. Learning complete lattices for manifold mathematical morphology. In Proc. of the ISMM'09, pages 1-4, 2009.

13. R. Lorand. Aesthetic order: a philosophy of order, beauty and art. Routledge studies in twentieth century philosophy. Routledge, 2000.

14. F. Meyer. The levelings. In Proc. of the ISMM'98, pages 199-206, Norwell, MA, USA, 1998. Kluwer Academic Publishers.

15. K. Muller, S. Mika, G. Ritsch, K. Tsuda, and B. Scholkopf. An introduction to kernel-based learning algorithms. IEEE Trans. on Neural Networks, 12:181-201, 2001.

16. L. Najman and H. Talbot. Mathematical morphology: from theory to applications. ISTEWiley, June 2010.

17. C. Ronse. Idempotent block splitting on partial partitions, i: Isotone operators. Order, 28:273306, 2011.

18. P. Salembier and J. Serra. Flat zones filtering, connected operators, and filters by reconstruction. IEEE Transactions on Image Processing, 4(8):1153-1160, 1995.

19. J. Serra. Image Analysis and Mathematical Morphology. Academic Press, Inc., Orlando, FL, USA, 1982.

20. J. Serra. Image Analysis and Mathematical Morphology, Vol. 2: Theoretical Advances. Academic Press, February 1988.

21. J. Serra. Tutorial on connective morphology. Selected Topics in Signal Processing, IEEE Journal of, 6(7):739 -752, nov. 2012.

22. P. Soille. Morphological Image Analysis. Springer-Verlag, 2003.

23. H. Talbot, C. Evans, and R. Jones. Complete ordering and multivariate mathematical morphology. In Proc. of the ISMM'98, pages 27-34, Norwell, MA, USA, 1998. Kluwer Academic Publishers.

24. S. Velasco-Forero and J. Angulo. Mathematical morphology for vector images using statistical depth. In Mathematical Morphology and Its Applications to Image and Signal Processing, volume 6671 of Lecture Notes in Computer Science, pages 355-366. Springer Berlin / Heidelberg, 2011. 
25. S. Velasco-Forero and J. Angulo. Supervised ordering in $\mathbb{R}^{p}$ : Application to morphological processing of hyperspectral images. IEEE Transactions on Image Processing, 20(11):33013308 , nov. 2011.

26. S. Velasco-Forero and J. Angulo. Random projection depth for multivariate mathematical morphology. Journal of Selected Topics in Signal Processing, 6(7):753-763, 2012.

27. S. Velasco-Forero and J. Angulo. Classification of hyperspectral images by tensor modeling and additive morphological decomposition. Pattern Recognition, 46(2):566-577, 2013. 